CAHIERS DE

NARRATOLOGIE

\section{Cahiers de Narratologie}

Analyse et théorie narratives

$36 \mid 2019$

Rhétorique et représentations de la culture mafieuse. Images, rituels, mythes et symboles

\title{
Discours mafieux, culture mafieuse
}

Manuela Bertone, Antonio Nicaso and Donato Santeramo

\section{(2) OpenEdition \\ Journals}

Electronic version

URL: http://journals.openedition.org/narratologie/9588

DOI: 10.4000/narratologie.9588

ISSN: 1765-307X

Publisher

LIRCES

\section{Electronic reference}

Manuela Bertone, Antonio Nicaso and Donato Santeramo, "Discours mafieux, culture mafieuse », Cahiers de Narratologie [Online], 36 | 2019, Online since 11 January 2020, connection on 24 September 2020. URL : http://journals.openedition.org/narratologie/9588 ; DOI : https://doi.org/10.4000/ narratologie.9588

This text was automatically generated on 24 September 2020.

Article L.111-1 du Code de la propriété intellectuelle. 


\title{
Discours mafieux, culture mafieuse
}

\author{
Manuela Bertone, Antonio Nicaso and Donato Santeramo
}

1 Il existe depuis toujours une sorte de mélange entre le crime (plus ou moins) organisé et ses représentations. Cet amalgame, qui créé une sorte d'écran fantasmatique sur lequel on peut projeter ses anxiétés, a gagné en vigueur et consistance à partir des années 1830 lorsque dans la presse ont commencé à apparaître des colonnes consacrées aux faits-divers, mais aussi des feuilletons et de véritables chroniques judiciaires ${ }^{1}$. La fondation en 1825 du quotidien La Gazette des Tribunaux, journal de jurisprudence et des débats judiciaires marque un véritable tournant dans ce domaine :

Journal quotidien, «La Gazette » se consacre exclusivement aux matières de droit, aux audiences des tribunaux, aux nouvelles judiciaires, c'est-à-dire à tout ce qui se rattache à l'étude ou à la pratique de la jurisprudence. On y trouve régulièrement des articles de fond sur des questions de droit ou des problématiques juridiques nouvelles : questions électorales, liberté de la presse, délits d'opinion, abolition de la peine de mort, amélioration des bagnes, régime des prisons, réforme du code pénal et du système pénitentiaire ${ }^{2}$.

2 Les histoires des « coquins » et des « bagnards » qui ravagent la France de la Monarchie de Juillet sont dès lors connues et commentées par le plus grand nombre. Les écrivains profitent à leur tour de cette manne d'informations : avec le Londres dickensien, Paris devient rapidement la capitale où se concentrent toutes les misères et la laideur du monde, grâce à la vitalité inépuisable d'auteurs comme Balzac, Sue ou Féval.

3 C'est justement un roman de Paul Féval, Les Habits Noirs (paru chez Hachette en 1863) qui démontre à quel point la transposition symbolique façonne et influence la compréhension de la réalité, car c'est bien à leur tenue que l'on "reconnaît » les membres d'une mystérieuse organisation criminelle secrète, capables de vols et trafics en tout genre, sans jamais être inculpés. Ce processus inconscient consistant à attribuer à un objet le sens d'un autre, jusqu'à ce qu'il le représente et le remplace complètement, s'avère également décisif pour l'enracinement de la Main Noire dans l'imaginaire populaire. «The Black Hand » n'est autre qu'une construction médiatique et sociale qui s'impose au Canada, aux États-Unis et en Australie à la fin du XIX ${ }^{e}$ siècle, transformant une technique d'extorsion somme toute banale, utilisée par des criminels d'origines diverses, en une organisation criminelle structurée (la « Mano Nera») aux 
ramifications internationales, rattachée à la mafia sicilienne. La source de cette transformation se trouve dans un procès tenu en 1884 en Espagne, au cours duquel l'expression "mano negra» est utilisée en référence à une présumée organisation secrète d'anarchistes. L'affaire sert alors au gouvernement libéral de Práxedes Mateo Sagasta pour étouffer les revendications du prolétariat agricole d'Andalousie en lutte contre les propriétaires terriens. Lors du procès, de fausses preuves sont fabriquées pour envoyer sept paysans à l'échafaud (huit autres seront condamnés à des peines de prison). Les nouvelles du procès font à l'époque le tour du monde.

Dans la première moitié du XIX ${ }^{e}$ siècle, il existe en Italie de nombreuses organisations criminelles qui ont les mêmes caractéristiques que les bandes françaises des coquins. À Turin, on appelle «còca » un gang de criminels qui agissent de concert avec d'anciens fonctionnaires de police. À Milan, les jeunes réunis dans la "compagnia della tèpa »se distinguent par le port d'un pull-over plumé et un chapeau tricorne. En Romagne sévit la bande à Stefano Pelloni, dit le «Passatore ». Et dans le Sud de la Péninsule surgissent et se déploient nombreux groupuscules sans nom. Seule la Camorra a une identité affirmée, puisqu'elle prend le nom de l'activité d'extorsion qui lui est propre: «fare camorra » [faire camorra] signifie en effet extorquer de l'argent (ou autre chose). Le premier à en raconter les faits et gestes est Alexandre Dumas, dans le cadre d'une série d'articles rédigés au printemps 1862, lors de son séjour à Naples. L'attention de l'écrivain se focalise sur les "classes dangereuses ", dont les occupations ne sont que dépravation morale et criminelle, et qui sont tout à fait distinctes de ce que l'on appelle les « classes laborieuses ", auxquelles appartiennent les ouvriers et les artisans, à savoir des gens honnêtes. Dotés d'une force d'évocation hors pair, ses textes ${ }^{3}$ finiront par influencer l'ensemble des récits à venir.

C'est un autre auteur français, le géographe Élisée Reclus qui, en 1865, allé à la rencontre des mystères de Palerme, découvre la "maffia", une sorte de camorra sicilienne, semblable à celle qu'a décrite Dumas ${ }^{4}$. Cette même «maffia » qui, deux ans auparavant, avait tiré son nom d'une œuvre théâtrale, Li mafiusi di la Vicaria, écrite par un comédien et directeur artistique, Giuseppe Rizzotto, avec l'aide d'un instituteur, Gaspare Mosca. C'est l'histoire d'un délinquant d'extraction populaire, arrogant et vulgaire, appartenant à une confrérie créée sur le modèle de la Camorra napolitaine. Rizzotto et Mosca s'inspirent de la description des prisons que donne Benedetto Naselli dans son ouvrage de 1852, I misteri di Palermo. Naselli décrit des détenus forcés de payer une sorte de taxe pour obtenir une meilleure couchette ou un petit espace de vie où ils ne seraient pas continuellement harcelés : une forme d'extorsion présentée comme une offre spontanée servant à alimenter l'huile d'une lampe votive ${ }^{5}$.

6 Même la 'Ndrangheta - aujourd'hui l'organisation criminelle la plus riche et la plus puissante - est dès le départ tributaire de la littérature. Son mythe fondateur s'inspire en effet d'un roman populaire paru en France en 1845, Les mystères de l'inquisition et autres sociétés secrètes d'Espagne, dont l'auteur, l'écrivaine-voyageuse allemande Irène de Suberwick, se cache derrière le pseudonyme Victor de Féréal ${ }^{6}$. Dans ce feuilleton à succès, là où il est question de la fantomatique Garduña, l'organisation espagnole qui serait à l'origine de la 'Ndrangheta, est narrée l'histoire de trois chevaliers qui, risquant leur vie, promettent de libérer une fille enlevée pour satisfaire les envies brutales de l'inquisiteur. Osso, Mastrosso et Carcagnosso, dans le mythe fondateur de la 'Ndrangheta, sont ces trois chevaliers qui, dans l'Espagne du XV siècle, se débarrassent d'un petit tyran afin de sauver l'honneur d'une jeune fille vulnérable. Contraints de fuir 
l'Espagne, ces trois braves se réfugient à Favignana, une des îles Egadi, au cœur de la Méditerranée. D'après la légende, c'est là qu'ils créent une organisation dont le but serait de protéger les faibles et les démunis contre les malversations des riches et des puissants. Osso décide de rester en Sicile, où il fonde la Mafia; Mastrosso se rend en Calabre, où il fonde la 'Ndrangheta; et Carcagnosso s'installe à Naples, où il fonde la Camorra $^{7}$.

Qu'il s'agisse des romans fleuves, de récits de voyage ou de pièces de théâtre, les criminels évoluent dans une sphère à part et sont l'indice d'une faiblesse de la société, mais aussi des individus organisés en associations criminelles soutenues et légitimées par le pouvoir en place, qui s'en sert à de fins politiques. En Sicile comme du reste en Calabre et en Campanie, les mafias sont le bras armé du pouvoir et par moments finissent par assurer l'ordre public, agissant sur le modèle de la haute police napoléonienne (on sait que des ministres de l'intérieur de l'empereur tels que Fouché et Savary engageaient des criminels pour combattre d'autres criminels) ${ }^{8}$. En 1860, le boss calabrais Raffaele Morgante est nommé chef de la garde municipale, tandis que le boss de la Camorra de Naples, Salvatore De Crescenzo, devient chef de la garde nationale. D'ailleurs, ces méthodes avaient été testées à l'époque des Bourbons, notamment lorsque le chef de la police Salvatore Maniscalco utilisait des malfaiteurs pour pourchasser des bandits, en Sicile. La littérature accompagne et représente ce phénomène: songeons encore à Balzac, à sa Comédie Humaine, où le personnage de Vautrin doit beaucoup à la personne d'Eugène-François Vidocq, l'ancien forçat devenu fondateur et premier directeur de la brigade de sûreté de Paris. La représentation littéraire des " classes dangereuses » établit solidement une idée simplifiée des mafias. Par ailleurs, elle contribue à raffermir l'identité de l'organisation criminelle en lui offrant la matière qui lui permet d'inventer sa propre tradition'.

Cependant, cela ne suffit pas à expliquer pourquoi les organisations criminelles perdurent malgré les imposantes ressources humaines et financières déployées pour les combattre. Qu'est-ce qui distingue le crime organisé des nombreuses bandes de malfaiteurs qui errent dans les villes, les banlieues et les villages du monde? Qu'est-ce qui fait que ces associations de malfaiteurs soient pour l'heure invincibles? Dans quelle mesure les autorités politiques, sociales et civiles sont-elles responsables de l'échec des différentes stratégies de lutte contre ce phénomène criminel ?

Rappelons tout d'abord que les mafias ont survécu à toutes les mutations sociales, en partie parce qu'elles sont parvenues à s'y adapter, mais surtout parce qu'elles ont su se présenter comme de véritables garants du passé, des traditions religieuses, des valeurs morales et sociales ponctuellement menacées par le changement, tout en intégrant et en exploitant les innovations qui ont vu le jour au fil du temps. C'est sans doute cette capacité à se positionner dans la modernité sans négliger les coutumes ancestrales, et toujours en tant que protecteurs des faibles, qui leur permet de s'enraciner partout, y compris loin de leur territoire d'origine.

10 L'invention du nom de l'organisation ou de ses chefs n'est qu'un début. Il est ensuite indispensable de créer une culture ou plutôt une «culture parallèle $»^{10}$ qui permette aux personnes impliquées d'avoir un statut social, un statut de haut rang s'entend, qui sera confirmé par la noblesses des gestes accomplis. Il faut donc échafauder un lien avec un passé prestigieux, non seulement pour justifier l'existence de l'organisation, mais aussi (ou surtout) pour qu'elle soit perçue comme une coterie bienveillante, capable de protéger des gens ordinaires contre toute sorte d'abus. C'est par cette image 
édifiante que l'on construit la légitimité de l'organisation et de ses membres : c'est tout l'intérêt de se doter d'un mythe fondateur d'envergure qui, concrètement, justifie toute action entreprise.

11 Le mythe, pour reprendre la définition de Giambattista Vico, est "vera narratio ", à savoir un récit vrai. Il s'agit de la narration d'une histoire dépourvue d'attaches avec la pensée spéculative ou rationnelle et malgré cela, puisqu'appliquée à la structure de l'existant, porteuse d'une forme de vérité et perçue comme "vraie». Le mythe joue donc un rôle fondamental dans la formulation de la cosmologie, de l'eschatologie et de la phénoménologie de l'univers, d'une nation, d'un peuple. Et aussi d'une organisation criminelle. Ce qui rend le mythe efficace, c'est qu'il n'est pas l'expression d'une chose ou d'un fait : il est la chose, il est le fait, en termes absolus. Autrement dit, à travers le mythe, c'est l'essence même d'une idée qui est offerte.

Quasiment toutes les organisations criminelles du monde utilisent des mythes fondateurs en rapport avec les grands récits qui sont à la base de la création d'empires et de nations. À ce propos, on peut citer Ab Urbe Condita de Tite-Live et l'Énéide de Virgile, deux ouvrages écrits quelques siècles après les événements qu'ils relatent, où la réalité côtoie la fiction et les traditions grecque et latine s'unissent pour construire et valoriser les nobles racines des lignées des Romains illustres. Les mythes plus récents exercent également une certaine fascination auprès des mafieux. Songeons par exemple à celui de la frontière américaine, de l'Occident extrême, dont la conquête assoit la suprématie des hommes blancs sur des régions sauvages et des populations sauvages: un mythe créé dans le but d'occulter la véritable histoire des colons et surtout le génocide organisé contre les autochtones. Ou à celui des Beati Paoli, qui charme, de nos jours encore, les mafieux siciliens, qui s'imaginent être les héritiers des membres de la société secrète du même nom, qui aurait été créée à Palerme au moyenâge pour défendre les droits des gens pauvres et des opprimés, persécutés aussi bien par l'Église que par l'État ${ }^{11}$. Sans oublier les Triades chinoises, qui prétendent que leurs origines remontent à un groupe de moines bouddhistes patriotes ayant lutté, au XVII siècle, pour libérer le pays d'un despote étranger. Ou le réseau japonais des Yakuza, qui retracent leurs origines à un groupe de samouraïs rebelles qui, au début du XVII ${ }^{\mathrm{e}}$ siècle, auraient défendu le peuple de l'empereur et son armée.

Même si le mythe ne pose et ne se pose pas la question de la distinction entre fiction et réalité, entre pensée intuitive et pensée rationnelle, sa fonction consiste justement à combler le manque de plénitude existentielle qu'engendre l'ordre des choses. C'est pourquoi il permet aux organisations criminelles de "justifier » non seulement leur existence mais aussi les actions de ceux qui s'y identifient. Sa puissance évocatrice est renforcée par l'usage systématique de rituels et de symboles qui servent aussi à satisfaire l'appétit d'images sacrées et de gestes solennels qu'éprouvent les adeptes des organisations criminelles. La participation à des cérémonies de type religieux ou pseudo-religieux permet d'incarner le mythe, d'effacer complètement la ligne de démarcation qui sépare la fiction de la réalité, les héros de la mythologie des malfaiteurs en chair et en os et, par conséquent, d'assurer une autorité soi-disant spirituelle aux organisations criminelles. Le rite d'initiation est l'indicateur anthropologique qui marque l'entrée de l'individu dans la sphère vécue du mythe: accepté dans un groupe, dans une société, il sort de l'adolescence, devient adulte, renaît pour commencer une nouvelle vie. Chez les mafieux, l'initiation implique aussi que l'on renonce au passé, à sa vie précédente. L'organisation criminelle est comparable à un 
ordre religieux : c'est une société de vie consacrée, où l'on prête serment, on s'adonne à des pratiques symboliques variées, on suit des règles de comportement, on accepte des punitions, bref, on évolue dans la contrainte assumée.

En fait, ce sont l'appropriation et le détournement de mythes des origines, symboles et rituels qui, au moins autant que l'argent et le pouvoir conquis, permettent aux mafias de perdurer, puisque la transmission de la «tradition » de génération en génération ne fait que la consolider et, partant, justifier et soutenir l'action de ceux qui se reconnaissent en elle. Les mafias en sont conscientes qui n'abandonnent ni récits ni mises en scène. Bien au contraire, elles se consacrent même à la modernisation et à l'actualisation des histoires qui les concernent, tout en préservant leurs qualités légendaires, par exemple en utilisant des groupes musicaux, souvent directement rémunérés par des chefs mafieux, pour décrire et célébrer la violence des clans. On est loin des mélodies d'autrefois qui racontaient la vie de brigands et autres malfaiteurs, sans toutefois en faire l'éloge ou en vanter les crimes. Aujourd'hui, dans le monde entier, il est question de " promotion » de la criminalité par la chanson, de «publicité » pour les bonnes actions des boss mafieux en faveur des quartiers dont ils auraient amélioré le niveau de vie (suivi par des millions de followers, le Movimiento Alterado mexicain-américain, avec ses narcocorridos qui racontent le quotidien des trafiquants de drogue, est l'un des plus performants) ${ }^{12}$.

Dans ce numéro des Cahiers de narratologie, nous avons souhaité réunir les contributions de plusieurs spécialistes de l'étude et du décryptage de ce phénomène criminel très particulier que sont les mafias, en nous focalisant, dans un souci de clarté et de cohérence, sur les trois principales mafias italiennes: Cosa Nostra, 'Ndrangheta, Camorra. Des organisations criminelles nées dans des régions de la Péninsule (la Sicile, la Calabre, la Campanie), mais dont les réseaux émaillent la planète entière, nous obligeant ainsi à questionner notre propre capacité à saisir et interpréter les messages de plus en plus nombreux et diversifiées qu'elles diffusent par nouveaux médias interposés. Sémioticiens, historiens, experts auprès de ministères ou de tribunaux, criminologues, critiques littéraires, spécialistes de l'information, du renseignement et de la communication, ils ont accepté de travailler ensemble sur un sujet qui correspond aux orientations actuelles de la recherche, un sujet proche de leurs intérêts personnels et de leur profession, qui s'inscrit aussi plus largement dans les préoccupations sociales et politiques de notre temps.

16 La rhétorique du discours mafieux et les représentations de la culture mafieuse, au cœur de ce volume monographique, seront examinées en prêtant une attention particulière aux images, rituels, mythes et symboles que les associations de malfaiteurs utilisent pour communiquer non seulement entre elles, mais avec le monde extérieur. Tirant parti d'un imaginaire partagé, les mafias ont exploité et exploitent des pratiques anciennes et d'anciens langages afin de construire et de transmettre des récits autoréférentiels qui se valident d'eux-mêmes. Souvent hybrides, c'est-à-dire basées sur la contamination de traditions, de codes et de genres différents, les histoires élaborées par les mafias ont fini par influencer nos propres représentations de la pègre. Qui plus est, et cela frôle le paradoxe, en consacrant une partie de notre production culturelle au monde du crime, nous avons nous-mêmes nourri le désir d'autoreprésentation des mafieux, impactant l'image que le milieu veut donner de lui-même ainsi que son propre storytelling. 
17 De cet écheveau de récits, de contre-narrations et de co-narrations, désormais très dense et complexe, grâce aux contributions réunies ici, nous avons tenté de trouver le fil conducteur ou, du moins, de démêler la masse, en abordant une série de questions spécifiques dont la teneur peut être synthétisée sous deux rubriques : a) la construction sémiotique mafieuse ; b) les productions culturelles dérivées de cette pratique.

18 S'agissant de construction sémiotique, on a d'abord pu vérifier que la mafia s'est appropriée de mythes et rites anciens afin de construire sa propre image sur des bases édifiantes ou tout au moins prestigieuses, car il s'avère que l'éthique chevaleresque et l'éthique maçonnique sont les véritables mères nourricières de l'éthique mafieuse. Dans une course incessante à l'émulation, des rôles et des modèles édifiants sont détournés et adaptés: les paladins de France, les chevaliers templiers, les patriotes carbonari, les frères francs-maçons, sont autant de personnifications éblouissantes que les mafieux exploitent pour se donner une aura de solide respectabilité voire de charme envoûtant. De ce fait, la religion n'est pas non plus exclue du giron mafieux. Franchissant sans complexes la ligne qui sépare l'attraction de l'usurpation, la sphère profane de la sphère sacrée, les mafias s'emparent de pratiques rituelles consolidées pour en utiliser les aspects solennels et les transformer en mystères d'un cérémonial personnalisé d'initiation de novices, de confirmation et d'onction d'adeptes, s'assurant qu'ésotérisme et exotérisme, entrelacés, préservent et en même temps suscitent l'envie de percer l'énigme de la doctrine criminelle.

19 Quant aux productions culturelles, si on sait que la représentation du crime a depuis des décennies la part belle au cinéma comme à la télévision et, plus récemment, en streaming et dans les séries diffusées en ligne, on ne réfléchit peut-être pas encore assez au fait que les mafias, elles, répondent bien volontiers aux suggestions des fictions qui les mettent en scène, en se plaçant sans complexes dans la dynamique de spectacularisation du crime initiée par les créateurs d'images. La mafia se donne désormais en spectacle en exploitant une vaste palette de moyens et de support de communication: l'art mural (graffiti, tag) ou l'art urbain, l'art corporel (tatouage, peinture, scarification) et les réseaux sociaux, la chanson neomelodica et le Darknet, dans une course à la diffusion de signes et signaux dont nous avons essayé d'identifier les émetteurs et les destinataires, de cerner les origines et les objectifs.

20 Face à une telle masse de signes et de discours (souvent obliques), la question de l'interprétation s'est posée à nous qui avons réuni ces contributions en même temps que celle de notre responsabilité. L'exégèse, on le sait, légitime son objet. Et le crime organisé, on le sait aussi, bénéficie d'une rente d'image inestimable grâce au surplus de visibilité qu'on lui confère en l'exposant comme objet de création ou en exhibant sa créativité $^{13}$. Comment éviter de contribuer au renforcement du mythe et de tous les stéréotypes qui forgent la narratio mafieuse, dès lors que l'on se focalise sur ses aspects inventifs et romanesques, même si ce n'est pas pour attiser la curiosité du public ? Tout d'abord en offrant à nos lecteurs une réflexion qui procède systématiquement à la démythification des performances inventives de l'organisation mafieuse : déconstruire ses artifices narratifs, démasquer ses faux symboles, décortiquer ses constructions rituelles, révéler ainsi la brutalité primaire de sa manière et de sa raison d'être, la gravité fondamentale, absolue, des événements dont les mafias sont les protagonistes. Ensuite en refusant et réfutant avec soin les représentations schématiques des contextes dans lesquels agissent les mafias. Il est sans doute plus « rassurant » de parler des actions mortifères des mafieux en montrant qu'il existe autour du crime une 
société criminogène, où la corruption est omniprésente et dépasse les frontières des organisations mafieuses, mais nous estimons que l'on déforme lourdement la réalité lorsqu'on prétend (comme le font pléthore d'auteurs et de médias) que la vie des institutions, de la politique, des citoyens n'est pas nécessairement plus propre et saine que la vie criminelle ${ }^{14}$. Si leur capacité à maitriser le territoire, leur relative stabilité, s'inscrivent en Italie dans une histoire nationale indéniablement marquée par l'instabilité et l'incertitude, il ne faudra pas oublier que l'emprise et le succès des organisations criminelles sur le territoire de la Péninsule et au-delà sont en partie liés à des simplifications pernicieuses. Ces simplifications qui, finalement, convertissent en légende ce qui n'est au sens figuré comme au sens propre, qu'une manière inauthentique d'exister.

\section{NOTES}

1. Cf. Francesco Benigno, La mala setta. Alle origini di mafia e camorra 1859-1878, Einaudi, Torino, 2015

2. Site de l'ENAP, Ecole Nationale de l'Administration Pénitentiaire. Lien URL: https:// www.enap.justice.fr/histoire/la-gazette-des-tribunaux-1825-1832.

3. Cf. Alexandre Dumas, La Camorra et autres récits de brigandage, texte établi, présenté et annoté par Claude Schopp, Librairie Vuibert, Paris, 2011.

4. Cf. Élisée Reclus, La Sicile et l'éruption de l'Etna en 1865 : récit de voyage (éd. 1866), Hachette-BNF, Paris 2016. Le récit a été initialement publié dans Le Tour du Monde, Nouveau Journal des Voyages, VII, n. 338, 1866.

5. Cf. Benedetto Naselli, I misteri di Palermo, I buoni cugini, Palermo, 2017 (édition originale F. Abbate, Palermo, 1852).

6. Victor de Féréal, Mystères de l'inquisition et autres sociétés secrètes d'Espagne, Boizard, Paris, 1845. La première traduction italienne, I Misteri dell'Inquisizione e altre società segrete di Spagna, est publiée à Paris dès 1847. À ce propos, cf. Nicola Gratteri - Antonio Nicaso, Dire e non dire. I dieci comandamenti della 'ndrangheta nelle parole degli affiliati, Mondadori, Milano, 2012.

7. Cf. Nicola Gratteri - Antonio Nicaso, Fratelli di sangue, Mondadori, Milano, 2008.

8. Cf. Hélène L'Heuillet, Basse politique, haute police. Une approche historique et philosophique de la police, Fayard, Paris, 2001.

9. Sur la question de "l'invention de la tradition" dans l'univers mafieux, voir Marcello Ravveduto, Lo spettacolo della mafia. Storia di un immaginario tra realtà e finzione, Edizioni Gruppo Abele, Torino, 2019.

10. Cf. Fernand Dumont, "Pour situer les cultures parallèles ", in Dumont, Fernand (dir.), Les Cultures parallèles, Montréal, et Québec, Leméac et Institut québécois de recherche sur la culture, coll. « Questions de culture ", 3, 1982, p. 15-34.

11. Pour d'amples approfondissements, voir l'article de Francesco Valerio Tumbarello, « Les Beati Paoli : genèse d'un mythe ", dans ce numéro des Cahiers de narratologie.

12. Cf. Claude Chastagner, «Les narcocorridos du Movimiento Alterado : une poésie de la rue?", Les Cahiers de Framespa [en ligne], 21, 2016. Lien URL : http://journals.openedition.org/framespa/ 3908 
13. Cf. Nicola Gratteri - Antonio Nicaso, L'inganno della mafia. Quando i criminali diventano eroi, RaiEri, Roma, 2017.

14. Cf. Nicola Gratteri - Antonio Nicaso, Padrini e padroni. Come la 'ndrangheta è diventata classe dirigente, Mondadori, Milano 2016.

\section{AUTHORS}

\section{MANUELA BERTONE}

Manuela Bertone, ancienne élève de Harvard University (AM 87, PhD 90), est professeur des universités en études italiennes à l'Université Côte d'Azur de Nice. Ses travaux scientifiques sont consacrés à Carlo Emilio Gadda, dont elle a publié I Littoriali del lavoro e altri scritti giornalistici 1932-1941, au roman et à l'histoire culturelle de l'Italie moderne et contemporaine. Elle a récemment contribué à l'ouvrage L'autre front/Il fronte interno. Art, culture et propagande dans les villes italiennes de l'arrière 1915-1918, qu'elle a codirigé (2018), et au numéro monographique de la revue « Europe » (2019) intitulé Giuseppe Tomasi di Lampedusa. Elle est associée au projet de recherche Noicontrolemafie, dirigé par Antonio Nicaso.

\section{ANTONIO NICASO}

Antonio Nicaso, journaliste, historien des organisations criminelles, est un expert mondialement connu du phénomène mafieux, notamment de la 'Ndrangheta, et travaille comme consultant des forces de police de nombreux pays. Il enseigne l'histoire sociale du crime organisé à la Queen's University de Kingston et est Associate Director du programme d'études italiennes à Middlebury College. Avec le juge Nicola Gratteri (actuel procureur de la République de Catanzaro), il est l'auteur de nombreux bestsellers consacrés aux mafias, traduits en plusieurs langues. Parmi les plus récents, on peut citer Oro bianco (2015), Fiumi d'oro (2017), L'inganno delle mafie (2017), La rete degli invisibili (2019). Son Business or Blood (2015) a inspiré la série Bad Blood, diffusée sur Netflix.

\section{DONATO SANTERAMO}

Donato Santeramo, après une laurea à l'Université de Rome La Sapienza, a obtenu un $\mathrm{PhD}$ à l'Université de Toronto. Professeur de littérature théâtrale, cinéma et Cultural Studies, il dirige le Department of Languages, Literatures and Cultures à la Queen's University de Kingston. Parmi ses ouvrages, on peut citer Luigi Pirandello: la parola, la scena e il mito (2007) et Il laboratorio teatrale pubblico di Edward Gordon Craig (2019). Auteur théâtral et metteur en scène, il a produit plusieurs pièces au Canada et aux États-Unis. Avec David Cronenberg, il a participé à la réalisation d'expositions et à la création de textes pour le cinéma. Il est associé au projet de recherche Noicontrolemafie, dirigé par Antonio Nicaso. 